\title{
Validación española de la escala de frustración de las necesidades psicológicas (EFNP) en el ejercicio físico
}

\author{
Alvaro Sicilia*, Roberto Ferriz y Piedad Sáenz-Álvarez \\ Universidad de Almería \\ (Recibido el 14 de Junio 2012; Aceptado el 21 de Noviembre 2012)
}

\begin{abstract}
RESUMEN: El objetivo de este estudio fue validar la versión española de la Escala de las Necesidades Psicológicas (EFNP) en el contexto del ejercicio físico. Una muestra de 509 estudiantes universitarios participaron en el estudio, analizando las propiedades psicométricas de la EFNP a través de diferentes análisis. Los resultados ofrecieron apoyo tanto a una estructura de tres factores relacionados (i.e., frustración de necesidad de autonomía, relación y competencia) como a un modelo de orden superior donde las tres subescalas conforman un constructo denominado frustración de las necesidades. La estructura de ambos modelos se mostró invariante por género. Se obtuvieron valores alfa de Cronbach iguales o superiores a .70 en las subescalas y unos adecuados niveles de estabilidad temporal. Además, los estudiantes que informaron mayores niveles de ansiedad físico-social obtuvieron puntuaciones más altas en las tres subescalas respecto al grupo que informó menores niveles de ansiedad físico-social. Los resultados de este estudio proporcionan evidencias de fiabilidad y validez de la EFNP en el contexto español.
\end{abstract}

Palabras clave: teoría de la autodeterminación, necesidades psicológicas, escala, propiedades psicométricas.

\section{Spanish validation of the psychological needs thwarting scale in exercise}

\begin{abstract}
The purpose of this study was to validate the Spanish version of the Psychological Needs Thwarting Scale (PNTS). A sample of 509 university students took part in this research and psychometric properties of PNTS were analyzed through different analysis. The results supported both a first-order three-factor model (i.e., thwarting of autonomy, relatedness and competence) and a higher-order model (i.e. three subscales conforming a construct named psychological need thwarting). The structure of both models was invariant across gender. Alpha values equal to o higher than .70 and suitable levels of temporal stability were obtained. Furthermore, students reporting higher levels of socialphysique anxiety had higher scores in all subscales than the group reporting lower levels of social-physique anxiety. The findings of this study provided reliability and validity for the PNTS in a Spanish context.
\end{abstract}

Keywords: self-determination theory, psychological needs, scale, psychometric properties. 


\section{INTRODUCCIÓN}

La investigación ha mostrado extensivamente que el ejercicio físico produce beneficios físicos y psicológicos positivos (Biddle y Fox, 1989; Glenister, 1996; Moustaka, Vlachopoulos, Kabitsis, y Theodorakis, 2012; Sallis y Owen, 1999). Sin embargo, la investigación ha sugerido también que el ejercicio físico puede estar asociado a experiencias y resultados negativos, tales como la dependencia al ejercicio (Hausenblas y Symons Downs, 2002), desordenes de la alimentación (Gargari, Khadem-Haghighian, Taklifi, Hamed-Behzad, y Shahraki, 2010), y trastornos afectivos como ansiedad, baja autoestima, depresión y estrés (Gargari et al., 2010; Salmon, 2001). En este sentido, es importante entender los factores contextuales que están detrás tanto de las experiencias negativas como positivas en el ejercicio físico. Basado en la teoría de la autodeterminación (TAD; Deci y Ryan, 1985), el objetivo de esta investigación fue validar al contexto español una escala que permita evaluar la frustración de las necesidades psicológicas en el ejercicio físico.

Desde hace décadas la TAD ha sido aplicada como una meta-teoría para el estudio de la motivación y el comportamiento humano, siendo la teoría de las necesidades psicológicas básicas (TNPB) una de sus mini teorías (Deci y Ryan, 2000; Ryan, 1995; Ryan y Deci, 2002). De acuerdo con la TNPB, las necesidades son definidas como nutrientes esenciales para el crecimiento, la integridad y la salud de todo ser viviente (Deci y Ryan, 2000). Extendiendo este razonamiento al mecanismo psicológico, Deci y Ryan han defendido la idea de que las personas se fundamentan al menos en tres tipos de nutrientes que son esencialmente necesarios para la integridad, el bienestar y el crecimiento personal. Estos nutrientes son las necesidades de autonomía, competencia y relación. La TNPB postula que los factores o situaciones que facilitan estas tres necesidades favorecerán el bienestar, mientras que los factores que frustran el desarrollo de estas necesidades deberían deteriorar el bienestar de la persona. Dentro del contexto del ejercicio, la autonomía se refiere a la necesidad de desarrollar las actividades que uno encuentra de interés, poder elegir y ser capaz de participar en el proceso de toma de decisiones. La competencia refleja la necesidad de sentirse capaz y observar que los objetivos planteados se consiguen con el tiempo. La relación con los demás se refiere a la necesidad de mantener una buena relación con otros y sentirse aceptado por ellos.

La investigación previa ha establecido empíricamente una clara asociación positiva entre la satisfacción de las necesidades psicológicas y el bienestar físico y psicológico en diferentes dominios de la vida, incluido el ejercicio físico (Gagné, Ryan, y Bargmann, 2003; Ntoumanis, 2012; Reis, Sheldon, Gable, Roscoe, Ryan, 2000; Wilson, Longley, Muon, Rodgers, y Murray, 2006). No obstante, la TAD reconoce que, además del bienestar y el crecimiento psicológico, las personas pueden desplegar patrones conductuales, cognitivos o afectivos que reflejen un funcionamiento no óptimo, reflejando así el lado más oscuro de su existencia (Pyszczynski, Greenberg, y Solomon, 2000; Ryan y Deci, 2000). En esta línea, 
más recientemente investigaciones basadas en la TAD han analizado las relaciones negativas entre la satisfacción de las necesidades psicológicas y diferentes conductas desadaptativas (Hodge, Lonsdale, y Ng, 2008; Thøgersen-Ntoumani y Ntoumanis, 2007; Thøgersen-Ntoumani, Ntoumanis, Cumming, y Chatzisarantis, 2011; Van den Broeck, Vansteenkiste, Witte, Soenens, y Lens, 2010). Con ello, este grupo de investigaciones ha comenzado a proporcionar evidencias indirectas que apoyarían la hipótesis de que la frustración de las necesidades tiene efectos negativos sobre la salud y el bienestar de las personas. Sin embargo, en este proceso algunos investigadores han cuestionado que una baja puntuación en la satisfacción de las necesidades (o el uso de estos ítems redactados de forma inversa) pueda recoger la naturaleza activa y la intensidad de la frustración de las necesidades de acuerdo con la TAD (Bartholomew, Ntoumanis, Ryan, Bosch, y Thøgersen-Ntoumani, 2011; Bartholomew, Ntoumanis, Ryan, y ThøgersenNtoumani, 2011; Brien et al., 2012). De hecho, Bartholomew, Ntoumanis, Ryan, y Thøgersen-Ntoumani han indicado que la satisfacción de las necesidades suelen ser medidas utilizando ítems que sólo recogen las experiencias psicológicas positivas (e.g., sentimientos de apoyo, aceptación, entendimiento), de tal modo que probablemente no capture los aspectos negativos de estas experiencias (e.g., sentimientos de rechazo, desprecio, conflicto) que, sin embargo, pueden estar ocurriendo cuando las necesidades están siendo frustradas. Como sugieren estos autores, la frustración de una necesidad no reflejaría simplemente que la percepción de la satisfacción de esa necesidad es baja o su insatisfacción alta, sino, además, indicaría la percepción de que la satisfacción de la necesidad está siendo obstruida o frustrada activamente dentro de un contexto determinado. Así, por ejemplo, una persona que práctica ejercicio físico podría sentirse incompetente en la actividad que realiza simplemente porque no tiene la necesaria habilidad para realizar la actividad, a pesar del ánimo que le brindan sus compañeros. Sin embargo, otro practicante de ejercicio podría sentirse incompetente porque sus compañeros y monitor se muestran excesivamente severos y críticos con él. La primera situación sería un claro ejemplo de una insatisfacción de la necesidad de competencia, mientras que la segunda reflejaría un ejemplo de frustración de la necesidad.

A raíz de de la falta de instrumentos para medir la frustración de las necesidades, Bartholomew, Ntoumanis, Ryan, y Thøgersen-Ntoumani (2011) desarrollaron y validaron un instrumento orientado a medir este constructo en el contexto deportivo. Para ello, los autores llevaron a cabo tres estudios con muestras independientes de deportistas con edades comprendidas entre los 12 y 17 años. El primer estudio buscó generar y proporcionar evidencias de validez de contenido de un conjunto de 21 ítems designados para medir la frustración de las necesidades psicológicas. El segundo estudio testó la estructura factorial de la escala y apoyó la validez de constructo de un modelo de tres factores y 12 ítems, el cual se mostró invariante respecto al género, tipo de deporte, nivel de competición y experiencia deportiva. No obstante, la fiabilidad para la frustración de la autonomía estuvo por debajo de .70 y la correlación entre los factores de frustración de la 
necesidad de competencia y la necesidad de relación fue alta (.85). Para subsanar estas limitaciones del instrumento, los autores modificaron la redacción de algunos ítems y sometieron el modelo a nuevos AFC en un último estudio. Con los cambios realizados, la estructura del modelo se mantuvo intacta y presentó buenos ajustes, mejorando las fiabilidades de los factores, cuyos valores oscilaron entre .77 y .82 . Sin embargo, la correlación entre la frustración de la necesidad de competencia y la necesidad de relación se mantuvo alta (.83), mientras que la correlación entre la frustración de la necesidad de competencia y la necesidad de autonomía incrementó con respecto al segundo estudio, pasando de .59 a .79 en este último estudio. Aún así, los autores propusieron mantener la estructura de tres factores, dado que este modelo mostró índices de ajustes mejores que un modelo de un solo factor y que diferentes modelos donde dos de los tres factores fueron unidos. Además, Bartholomew, Ntoumanis, Ryan, y Thøgersen-Ntoumani mostraron evidencias de validez predictivas del instrumento, demostraron que la frustración de las necesidades era un predictor mejor de consecuencias negativas (e.g., agotamiento físico y emocional) que la satisfacción de las necesidades.

En líneas con el estudio anterior, Bartholomew, Ntoumanis, Ryan, Bosch et al. (2011) expandieron su estudio inicial examinando los efectos de la frustración de las necesidades psicológicas en el deporte sobre un número más amplio de resultados psicológicos, afectivos y conductuales desadaptativos para el desarrollo personal (e.g., depresión, transtornos de la alimentación, burnout, afecto negativo, mayores niveles de S-IgA). Los resultados de este estudio reforzaron la hipótesis de la TAD que la frustración de las necesidades presenta efectos empíricos significativos e independientes más allá de la insatisfacción de las necesidades. Estos resultados han sido reforzados recientemente en el ámbito del deporte (Mallinson y Hill, 2011), pero también en otros dominios de la vida, tales como el trabajo (Gillet, Fouquereau, Forest, Brunault, y Colombat, 2011).

A partir del estudio realizado por Bartholomew, Ntoumanis, Ryan y Thøgersen-Ntoumani (2011) se dispone de un instrumento que posibilita la medición de la frustración de las necesidades psicológicas de una forma más directa que con la escala de satisfacción de las necesidades. Hasta la fecha, los estudios que mostraban una relación negativa entre procesos desadaptativos y la satisfacción de las necesidades psicológicas (Vlachopoulos y Michailidou, 2006) no ofrecían suficiente evidencia de que estas necesidades estuvieran frustradas. El instrumento desarrollado por Bartholomew, Ntoumanis, Ryan y Thøgersen-Ntoumani permitirá analizar en futuras investigaciones las consecuencias distintas que puede tener la frustración de las necesidades psicológicas en contextos como el deportivo, la educación física y el ejercicio físico.

El objetivo de este estudio fue confirmar la estructura factorial de la Escala de la Frustración de las Necesidades Psicológicas (EFNP; Bartholomew, Ntoumanis, Ryan, y Thøgersen-Ntoumani, 2011) en el contexto español, a través del análisis de su validez de constructo por medio de la correlación de las subescalas y consistencia interna, su estabilidad temporal y su invarianza respecto al género. Como la EFNP puede ser usada para calcular tanto la puntuación de cada 
subescala como la puntuación media total (e.g., para integrarla en un modelo de ecuaciones estructurales complejo), el objetivo fue analizar ambas estructuras. En el primer modelo se hipotetizó que los tres factores de primer orden (i.e., satisfacción de la necesidades de autonomía, competencia y relación), estarían correlacionados. En el segundo modelo, se propuso una estructura con tres factores de orden primario y un segundo factor de orden superior (i.e., frustración de las necesidades).

Un objetivo secundario en este estudio fue mostrar evidencias de validez de criterio de la EFNP, a partir del análisis de las diferencias en las puntuaciones de la frustración de las necesidades, de acuerdo con el nivel de ansiedad físico-social de los participantes. La ansiedad físico-social ha sido asociada negativamente con la satisfacción de las necesidades psicológicas (Brunet y Sabiston, 2009; Thøgersen-Ntoumani y Ntoumanis, 2007). Sin embargo, hasta la fecha no se ha contado con un instrumento que mostrara evidencias directas de la asociación entre la frustración de las necesidades y la ansiedad física-social, a pesar de que la investigación ha demostrado que la frustración de las necesidades tiende a mostrarse como un predictor más fuerte de consecuencias desadaptativas para la persona que la insatisfacción de las necesidades (Barhtolomew, Ntoumanis, Ryan, Bosch, et al., 2011; Bartholomew, Ntoumanis, Ryan, y Thøgersen-Ntoumani, 2011). En base a las investigaciones previas, se hipotetizó que los estudiantes que informaran mayor nivel de ansiedad físico-social presentarían mayores puntuaciones en la frustración de las tres necesidades psicológicas.

\section{MÉTODO}

\section{Participantes}

Los participantes en este estudio fueron 509 estudiantes universitarios (330 hombres y 179 mujeres), con edades comprendidas entre los 18 y 38 años $(M=$ 22.02, $D T=3.20$ ), quienes manifestaron realizar ejercicio físico de forma habitual. En concreto, 247 participantes informaron realizar actividad física entre uno y tres días a la semana y 262 manifestaron realizar actividad física más de tres días a la semana. La media de ansiedad físico-social de los participantes fue de $2.39(D T=.83)$.

\section{Medidas}

Escala de la Frustración de las Necesidades Psicológicas en el ejercicio físico (EFNP). Se empleó una la versión adaptada y traducida de la Psychological Need Thwarting Scale (PNTS) de Bartholomew, Ntoumanis, Ryan, y ThøgersenNtoumani et al. (2011) para el deporte. La escala está precedida por la frase "En mi ejercicio físico...", y compuesta por doce ítems que se distribuyen en cuatro ítems para cada una de las tres subescalas en que está compuesta: a) frustración de la necesidad de autonomía (e.g., "me siento obligado u obligada a seguir las 
decisiones de los demás"); b) frustración de la necesidad de competencia (e.g., "hay situaciones en las que me siento incapaz") y c) frustración de la necesidad de relación (e.g., "siento que no le gusto a otras personas"). Para cada ítem los participantes deben indicar su respuesta en una escala Likert de 7 puntos, oscilando desde el 1 (totalmente en desacuerdo) al 7 (totalmente de acuerdo). Mayores puntuaciones indican más nivel de frustración de las necesidades psicológicas.

Escala de Ansiedad Físico-Social (SPAS-7). Se utilizó la versión en español (Sáenz-Alvarez, Sicilia, González-Cutre, y Ferriz, en prensa) del modelo unidimensional de la Social Physique Anxiety Scale establecido por Motl y Conroy (2000, 2001). Este instrumento contiene siete ítems (e.g., "hay ocasiones en que estoy molesto/a porque pienso que otros están evaluando mi peso o forma física negativamente"). Los participantes debían indicar su respuesta por medio de una escala Likert del 1 (nunca) al 5 (siempre). Mientras más elevadas sean las puntuaciones más alto se revelan los síntomas de ansiedad físico-social, salvo para el ítem 5 que está redactado de forma inversa (e.g., "me siento a gusto de cómo los demás aprecian la forma de mi cuerpo").

La versión española de la escala (Sáenz-Alvarez et al., en prensa) ha mostrado índices de ajuste apropiados en una muestra de adolescentes: $\chi^{2}(14, N=$ $398)=45.91, p<.001 ; \chi^{2} / g l=3.28$; CFI $=.98$; TLI $=.96$; IFI $=.98$; RMSEA $=.076(\mathrm{IC} 90 \%=.052-.101) ; \mathrm{SRMR}=.032)$. La estructura factorial del modelo se reveló también invariante respecto al género. Además, el instrumento mostró una adecuada consistencia interna (valor alfa de Cronbach $=.85$ ) y una elevada estabilidad temporal $(\mathrm{ICC}=.82)$. No obstante, dado que en este estudio los participantes fueron universitarios se ha procedido a testar la estructura del instrumento con un nuevo AFC. Los índices de ajuste del modelo fueron excelentes: $\chi^{2}(15, N=509)=22.56, p<.05 ; \chi^{2} / g l=1.74 ; \mathrm{CFI}=.99 ; \mathrm{TLI}=.99$; IFI $=.99 ; \mathrm{RMSEA}=.038 ; \mathrm{SRMR}=.024)$. Además, en este estudio el valor alpha de Cronbach para la SPAS-7 fue .85 .

\section{Procedimiento}

Con el fin de validar la escala al contexto español se empleó la estrategia de traducción inversa de Hambleton (1996). Para este proceso, la escala fue transcrita inicialmente al español por un grupo de traductores, mientras que posteriormente otro grupo tradujo la misma a su idioma original. Este proceso aseguró que la traducción respetara la coincidencia de la versión original de la escala. La versión resultante fue analizada por un grupo de tres expertos en ciencias de la actividad física y la psicología (Lynn, 1986), que consideraron adecuados los ítems que fueron validados para medir cada uno de los tres constructos, así como la correcta redacción de cada uno de ellos.

Una vez traducida la escala, se contactó con tres universidades pertenecientes a tres ciudades españolas para solicitarles su colaboración en el estudio. Antes de administrar el cuestionario a la totalidad de los participantes, éste fue cumplimentado por un pequeño grupo de alumnos universitarios para asegurarnos que 
entendían todos los ítems. La escala se administró en presencia de alguno de los miembros del grupo de investigación, que explicó la forma de cumplimentar la escala y resolvió las dudas que pudieron surgir durante el proceso de recogida de datos. La participación fue voluntaria y los participantes necesitaron aproximadamente 15 minutos para cumplimentar la escala.

\section{Análisis de datos}

Se realizó un análisis inicial de las propiedades psicométricas de la EFNP para el ejercicio físico que llevó a determinar la validez y fiabilidad de la escala para el contexto español. Para tal fin, se calcularon los estadísticos descriptivos, correlaciones bivariadas, análisis factorial confirmatorio (AFC) a partir del modelo de tres factores de la frustración de las necesidades psicológicas, un análisis de invarianza por sexo, y un análisis de consistencia interna. Además, para comprobar la estabilidad temporal de la escala, se realizó un r-test con una muestra independiente de estudiantes universitarios. Finalmente, se realizó un análisis de varianza para examinar la relación entre la frustración de las necesidades psicológicas y la ansiedad físico social. Para realizar los análisis estadísticos se utilizó el SPSS 20 y el AMOS 19.

Puesto que el coeficiente de Mardia fue alto (102.81), para los diferentes AFC se utilizó el método de máxima verosimilitud junto con el procedimiento de bootstrapping. Los estimadores no se vieron afectados por la falta de normarlidad, por lo que fueron consideramos suficientemente robustos (Byrne, 2001). Para tratar de aceptar o rechazar los diferentes modelos testados, se empleó una combinación de varios índices de ajuste: $\chi^{2} / g l$, CFI (Comparative Fit Index), TLI (Tucker Lewis Index), IFI (Incremental Fit Index), RMSEA (Root Mean Square Error of Approximation) más su intervalo de confianza al 90\%, y SRMR (Standardized Root Mean Square Residual). Dado que el $\chi^{2}$ es muy sensible al tamaño muestral (Jöreskog y Sörbom, 1993), se empleó el $\chi^{2} / g l$, el cual se considera aceptable con valores inferiores a 5 (Bentler, 1989). Los índices incrementales (CFI, TLI e IFI) muestran un buen ajuste con valores de .90 o superiores (Schumacker y Lomax, 1996), mientras que los índices de error se consideran aceptables con valores iguales o menores de .08 para RMSEA y SRMR (Browne y Cudeck, 1993; Hu y Bentler, 1999).

\section{RESULTADOS}

\section{Estadísticos descriptivos y correlaciones bivariadas entre los tres factores}

Los participantes revelaron puntuaciones por debajo del valor medio de la escala en los tres factores de la EFNP, siendo la puntuación más elevada para la frustración de la competencia $(M=2.12)$ y la más baja para la frustración de la relación $(M=1.79)$. El análisis correlacional de Pearson reveló una relación positiva y significativa $(p<.001)$ entre los tres factores, con valores que fueron 
entre .54 y .63. Las correlaciones entre cada uno de los factores y la medida global de frustración de las necesidades oscilaron entre .84 y .89 .

Tabla 1. Estadísticos Descriptivos y Correlaciones entre todos los Factores de la EFNP

\begin{tabular}{|c|c|c|c|c|c|c|c|c|}
\hline Factores & $M$ & $D T$ & Rango & Asimetría & Curtosis & 1 & 3 & 4 \\
\hline 1. Frustración necesidad autonomía & 1.81 & .83 & $1-7$ & 1.42 & 2.43 & $.63 * * *$ & $.54 * * *$ & $.84 * *$ \\
\hline 2. Frustración necesidad competencia & 2.12 & .86 & $1-7$ & 1.01 & .81 & & $.63 * * *$ & $.89 * *$ \\
\hline 3. Frustración necesidad relación & 1.79 & .85 & $1-7$ & 1.23 & 1.18 & & & $.84 * *$ \\
\hline 4. Frustración necesidades & 1.91 & .75 & $1-7$ & 1.10 & 1.34 & & & \\
\hline
\end{tabular}

$* * * p<.001$

\section{Análisis factorial confirmatorio}

Los resultados del modelo de tres factores y doce ítems (ver Figura 1) presentaron unos índices de ajuste aceptables: $\chi^{2}(51, N=509)=191,63, p<.001$; $\chi^{2} / g l=3.76$; CFI $=.92$; TLI $=.90$; IFI $=.92$; RMSEA $=.074$ (IC 90\% $=.063-$ $.085)$; SRMR $=.052$. Los pesos de regresión de los ítems oscilaron entre $.46 \mathrm{y}$ .81 , siendo todos ellos estadísticamente significativos $(p<.001)$. No obstante, se obtuvieron unas correlaciones elevadas entre los factores de frustración de la autonomía y frustración de la competencia (.89), y entre ésta última y la frustración de la relación (.87), mientras que entre la frustración de la autonomía y la frustración de la relación se obtuvo una correlación de .76. 


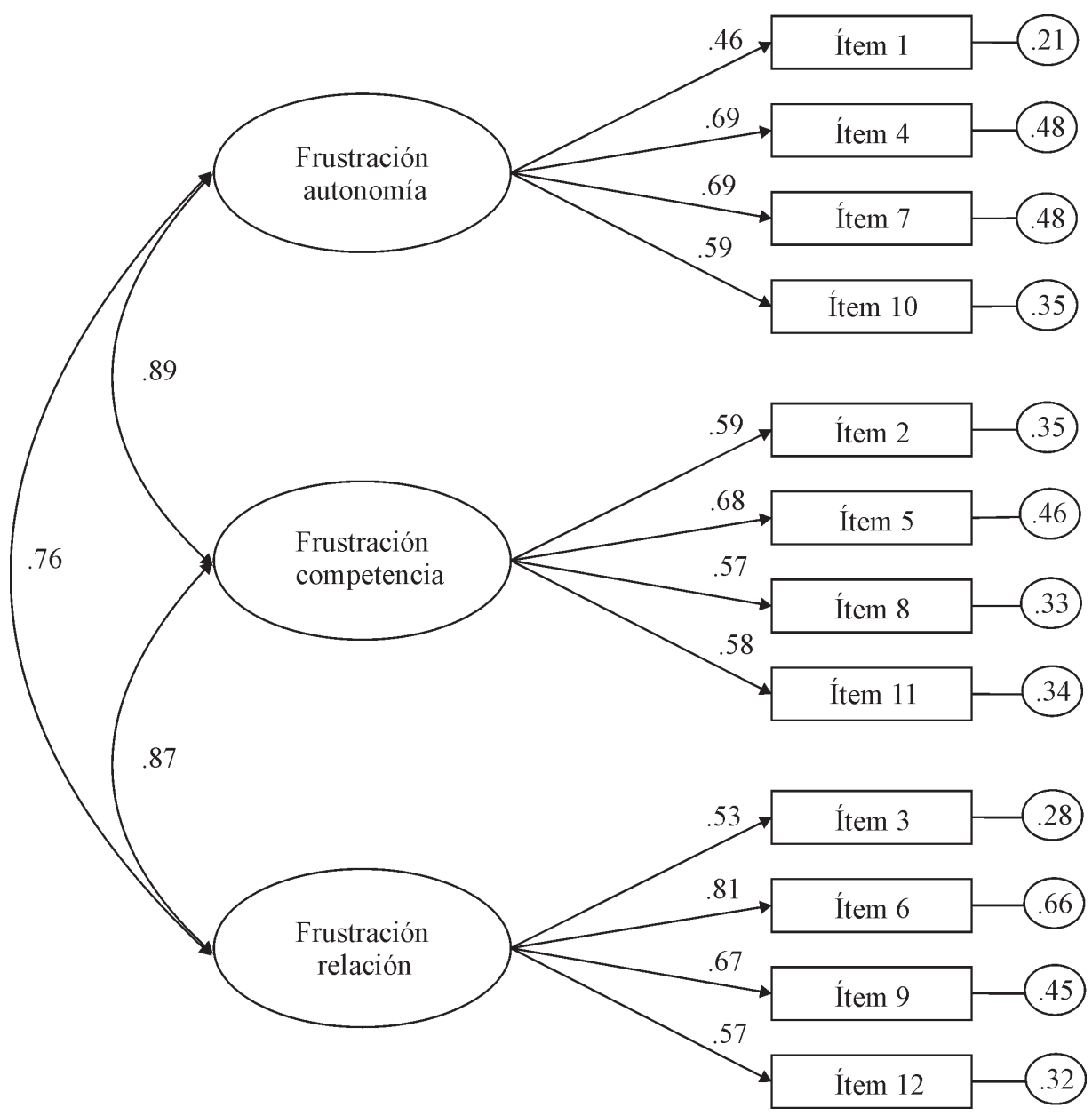

Figura 1. Análisis factorial confirmatorio de la EFNP. Las elipses representan los factores y los rectángulos representan los diferentes ítems. Las varianzas residuales se muestran en los círculos pequeños.

Debido a la alta correlación de los factores, la sostenibilidad de tres modelos alternativos con dos factores fue testada. En cada uno de estos modelos, dos de las subescalas fueron combinadas para formar un factor que se uniera al modelo junto con la subescala restante. Finalmente, un modelo de un solo factor, que aglutinaba a los doce ítems, fue también testado. Como puede observarse en la Tabla 2, tanto los modelos de dos factores como el modelo de un solo factor mostraron un ajuste peor en comparación con el modelo de tres factores. Unos mejores índices de ajuste del modelo de tres factores frente a modelos alternativos, donde se agrupan dos o más factores, apoya la validez discriminante de las subescalas. 
Tabla 2. Índices de Ajuste para los Modelos Factoriales Alternativos

\begin{tabular}{|c|c|c|c|c|c|c|c|c|}
\hline Modelos & $\chi^{2}$ & $g l$ & $\chi^{2} / g l$ & CFI & TLI & IFI & SRMR & $\begin{array}{l}\text { RMSEA } \\
\text { (IC 90\%) }\end{array}$ \\
\hline F1 $=$ Competencia/Autonomía $\quad F 2=$ Relación & $211.93 * * *$ & 53 & 4.00 & .91 & .89 & .91 & .053 & $.077(.066-.088)$ \\
\hline $\mathrm{F} 1=$ Autonomía/Relación $\mathrm{F} 2=$ Competencia & $250.71 * * *$ & 53 & 4.73 & .89 & .86 & .89 & .054 & $.086(.075-.097)$ \\
\hline F1 $=$ Competencia/Relación $F 2=$ Autonomía & $222.04 * * *$ & 53 & 4.19 & .91 & .88 & .91 & .052 & $.079(.069-.090)$ \\
\hline F 1 = Autonomía/Competencia/Relación & $255.23^{* * *}$ & 54 & 4.73 & .89 & .86 & .89 & .054 & $.060(.051-.069)$ \\
\hline
\end{tabular}

Los índices de ajuste para un modelo de orden superior (tres factores de primer orden y un factor de segundo orden denominado frustración de necesidades) se mostraron similares al modelo de tres factores, siendo sus valores aceptables: $\chi^{2}(51, N=509)=191.63, p<.001 ; \chi^{2} / g l=3.76 ;$ CFI $=.92 ;$ TLI $=.90 ;$ IFI $=.92$; $\mathrm{RMSEA}=.074(\mathrm{IC} 90 \%=.064-.085) ; \mathrm{SRMR}=.052$. En este modelo, todos los pesos de regresión estandarizados fueron significativos $(p<.001)$, siendo de .88 para frustración de autonomía, 1.01 para frustración de competencia, y .86 para frustración de relación.

\section{Análisis de invarianza}

Un análisis de invarianza multigrupo respecto al género fue realizado tanto para el modelo de tres factores primarios como para el modelo de orden superior de la EFNP. En la Tabla 3 se muestran los diversos índices de ajuste para los cuatro modelos comparados dentro de la primera estructura analizada (tres factores de primer orden). Diferencias significativas en el estadístico $\chi^{2}$ fueron encontradas entre el modelo no constreñido (Modelo 1) y los modelos con pesos de medida invariante (Modelo 2), covarianzas estructurales invariantes (Modelo 3), y medidas residuales invariantes (Modelo 4). En la Tabla 3 también se muestran los diversos índices de ajuste para los seis modelos comparados dentro de la estructura de un factor de orden superior. Igualmente, diferencias significativas fueron encontradas entre el modelo no constreñido (Modelo 1) y los modelos con pesos de medidas invariantes (Modelo 2), pesos estructurales invariantes (Modelo 3), covarianzas estructurales (Modelo 4), residuales estructurales invariantes (Modelo 5), y medidas residuales invariantes (Modelo 6). No obstante, dado que el coeficiente $\chi^{2}$ es sensible al tamaño de la muestra, se usó el criterio establecido por Cheung y Rensvold (2002) respecto al $\Delta$ CFI. De acuerdo con estos autores, valores del $\Delta$ CFI menores o iguales a .01 indica que la invarianza de la hipótesis nula no debería ser rechazada. De acuerdo a este criterio, los resultados apoyan la existencia de invariancia respecto al género tanto en el modelo de tres factores de orden primario como en el modelo de un factor de orden superior. 
Tabla 3. Análisis Multigrupo de Invarianza por Género

Modelo de tres factores de orden primario

\begin{tabular}{lllllllll}
\hline Modelos & $\chi^{2}$ & $g l$ & $\chi^{2} / g l$ & $\Delta \chi^{2}$ & $\Delta g l$ & CFI & IFI & SRMR RMSEA (IC 90\%)
\end{tabular}

$\begin{array}{lllllllllll}\text { Modelo } 1 & 263.49 & 102 & 2.58 & - & & - & .913 & .915 & .051 & .056(0.48-0.64)\end{array}$

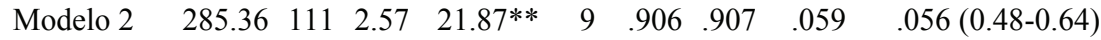

$\begin{array}{llllllllll}\text { Modelo } 3 & 304.78 & 117 & 2.60 & 41.29 * * * & 15 & .899 & .900 & .064 & .056(0.49-0.64)\end{array}$

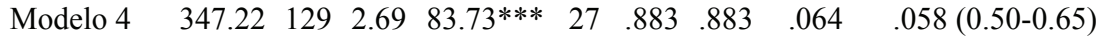

Modelo de un factor de orden superior

Modelos $\quad \chi^{2} \quad g l \quad \chi^{2} / g l \quad \Delta \chi^{2} \quad \Delta g l$ CFI $\quad$ IFI $\quad$ SRMR RMSEA (IC 90\%)

$\begin{array}{lllllllllll}\text { Modelo } 1 & 263.49 & 102 & 2.58 & - & - & .913 & .915 & .051 & .056 & (.048-.064)\end{array}$

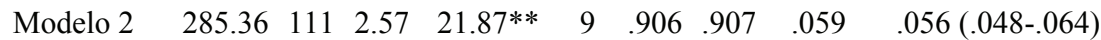

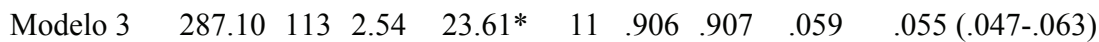

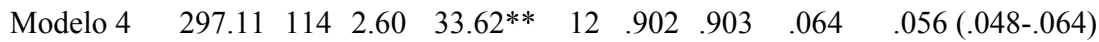

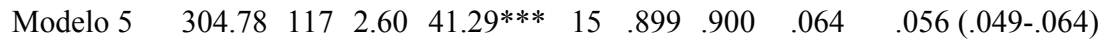

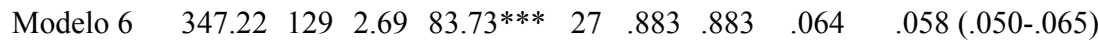

$* p<.05 ; * * p<.01 ; * * * p<.001$

\section{Análisis de fiabilidad}

Para determinar la fiabilidad de la escala se procedió a realizar un análisis de consistencia interna y un análisis de estabilidad temporal. En el primer caso, el análisis de consistencia interna reveló un valor alfa de Cronbach de .70 para la frustración de la autonomía, .70 para la frustración de la competencia, y .71 para 
la frustración de la relación.

Para analizar la estabilidad temporal de la escala, se utilizó una muestra independiente de 42 estudiantes universitarios con edades entre 20 y 31 años $(M=$ 22.00 DT = 2.92). Todos los participantes manifestaron practicar ejercicio físico de forma regular. La escala fue administrada dos veces, con un intervalo de tiempo de cuatro semanas entre la primera y segunda toma de datos. Para calcular la estabilidad temporal obtuvimos el coeficiente de correlación intra-clase (CCI) para cada una de las subescalas. Las medias para la frustración de la necesidad de autonomía fueron $2.07(D T=.89)$ y $1.95(D T=.91)$ con un CCI de $.90(\mathrm{IC}=.81$ .95). Para la frustración de la necesidad de competencia las medias fueron 2.18 $(D T=1.13)$ y $2.19(D T=1.17)$ con un CCI de $.92(\mathrm{IC}=.84-.92)$. Para la frustración de la necesidad de relación las medias fueron $1.99(D T=1.05)$ y $2.17(D T=$ 1.15) con un CCI de .85 (IC = .72-.92). Por lo tanto, los resultados muestran altos niveles de estabilidad temporal en las tres subesclas que componen la EFNP.

\section{Análisis de validez de criterio}

Para analizar las diferencias en la frustración de las tres necesidades psicológicas en función del nivel de ansiedad físico-social de los participantes, se utilizaron dos grupos, considerando el punto medio de la escala de ansiedad físicosocial. El primer grupo estuvo formado por 373 estudiantes, quienes puntuaron por debajo del punto medio de la escala en ansiedad físico-social, mientras el segundo grupo, formado por 136 participantes, informaron puntuaciones más altas de ansiedad.

El ANOVA en función de la ansiedad físico-social (ver Tabla 4) reveló diferencias significativas en los tres factores de la EFNP, así como en el valor global de frustración de las necesidades. Los estudiantes con mayor nivel de ansiedad físico-social obtuvieron mayores puntuaciones tanto en los tres factores como en el valor global de frustración de las necesidades psicológicas, comparado con el grupo que informó menores niveles de ansiedad físico-social.

Tabla 4. Análisis de Varianza de la Frustración de las Necesidades Psicológicas por Nivel de Ansiedad Físico-Social

\begin{tabular}{|c|c|c|c|c|c|c|}
\hline & \multicolumn{2}{|c|}{ Baja AFS } & \multicolumn{2}{|c|}{ Alta AFS } & \multirow{2}{*}{$F(1-507)$} & \multirow{2}{*}{$\eta^{2}$ parcial } \\
\hline & $M$ & $D T$ & $M$ & $D T$ & & \\
\hline Frustración autonomía & 1.70 & .79 & 2.10 & 87 & $24.73 * *$ & .34 \\
\hline Frustración competencia & 1.93 & .85 & 2.62 & 1.06 & $57.93 * *$ & .36 \\
\hline Frustración relación & 1.67 & .76 & 2.13 & .98 & $30.60 * *$ & .28 \\
\hline Fustración NPB & 1.77 & .67 & 2.29 & .82 & $51.85^{* *}$ & .18 \\
\hline
\end{tabular}




\section{DISCUSIÓN}

El objetivo de este estudio fue examinar la validez factorial, la consistencia interna, estabilidad temporal y validez de criterio de la EFNP. Los resultados de este estudio apoyaron la EFNP como un instrumento válido y fiable para evaluar en España la frustración de las necesidades en el contexto del ejercicio físico. A partir de ahora este instrumento podría ayudar a extender la investigación sobre las necesidades psicológicas, especialmente al permitir profundizar en el lado más oculto del desarrollo humano de acuerdo con la teoría de la autodeterminación (Ryan y Deci, 2000). De especial interés en esta línea puede ser la evaluación de la frustración de las necesidades como un predictor de consecuencias negativas para la salud y el desarrollo de la persona (Bartholomew, Ntoumanis, y Thøgersen-Ntoumani, 2011; Ntoumanis, 2012; Ryan y Deci, 2000).

Los resultados de este estudio revelaron que la estructura factorial de la EFNP mantiene para el contexto español el modelo de tres factores (i.e., frustración de la autonomía, competencia y relación), de acuerdo con los postulados de la TAD. Los análisis de fiabilidad apoyaron tanto la consistencia interna como la estabilidad temporal de las tres subescalas. Sin embargo, como ya ocurriera en estudios anteriores (Bartholomew, Ntoumanis, Ryan, y Thøgersen-Ntoumani, 2011), el modelo de tres factores de la EFNP mostró altas correlaciones entres sus subescalas. La validez discriminante de las subescalas fue apoyada al comparar este modelo con otros posibles modelos de dos y un solo factor, mostrando que la frustración de las tres necesidades representan constructos diferentes aunque correlacionados. La alta correlación entre las tres necesidades ha sido además encontrada previamente en estudios que analizan la satisfacción de las necesidades psicológicas tanto dentro como fuera de España (Luján y Ferriol, 2011; Moreno-Murcia, Huéscar, y Cervelló, 2012; Vlachopoulos y Michailidou, 2006). Como ha sugerido Bartholomew, Ntoumanis, Ryan y Thøgersen-Ntoumani, una posible explicación de este patrón podría venir dado por el hecho de que las tres necesidades suelen venir afectadas por factores o antecedentes comunes. Así, del mismo modo que factores o conductas que promueven la autonomía implican un reconocimiento o valoración de la persona que contribuyen posiblemente también a reforzar las relaciones y la competencia de uno, conductas o contextos excesivamente controladores pueden frustrar las tres necesidades en el sentido de que no sólo deteriora el sentimiento de autonomía, sino a menudo también devalúa la confianza en la persona y en su falta de habilidad.

La estructura de un modelo de orden superior, denominado frustración de necesidades, fue también apoyada a través de AFC. Este modelo es especialmente útil porque apoya el uso en la investigación de un valor global compuesto por la media de las tres subescalas. Metodológicamente este uso puede ser útil cuando el valor de la frustración de las tres necesidades se quiere considerar como un solo constructo dentro de un modelo más complejo donde se relacione con otros constructos. Teóricamente viene además justificado por la investigación previa que ha sugerido que las tres necesidades tienden a funcionar "al unísono en situa- 
ciones naturales" (Gagné et al., 2003, p. 386).

Los análisis multigrupo desarrollados evidenciaron que tanto la estructura de un modelo de tres factores como un modelo de orden superior de la EFNP fueron invariantes respecto al género. Este resultado es importante, dado que posibilita en futuros estudios la comparación de medias entre los hombres y mujeres. No obstante, estas investigaciones deberían examinar la invarianza de la EFNP respecto a otras variables. Por ejemplo, la invarianza por edad en este estudio no ha sido analizada debido a que la muestra de estudiantes representó poca variabilidad. Sin embargo, estudios futuros podrían examinar la invarianza respecto a diferentes grupos de edad teniendo en cuenta la división teórica que considera diferentes etapas en la vida: adolescente, jóvenes, adultos y personas mayores.

Finalmente, se encontraron evidencias para la validez predictiva de la escala. En concreto, el ANOVA mostró que los estudiantes que informaron mayores niveles de ansiedad físico-social puntuaron más alto tanto en el valor global de frustración como en cada una de las tres subescalas de la EFNP. Estos resultados se muestran en línea con los estudios previos que han mostrado una relación negativa entre la satisfacción de las necesidades psicológicas y la ansiedad físico-social (Brunet y Sabiston, 2009; Thøgersen-Ntoumani y Ntoumanis, 2007). Además, esta relación parece apoyar la idea planteada por Deci y Ryan (2000), de que la frustración de las necesidades psicológicas puede llevar a una búsqueda de necesidades substitutivas o compensatorias que realmente no satisfagan las necesidades frustradas pero que, sin embargo, proporcionen una satisfacción colateral. Así, si la necesidad que los estudiantes tienen de relacionarse durante su ejercicio físico es substancialmente frustrada, éstos podrían quererla compensar intentado ganar la aprobación de los demás y aumentar su valoración ante el grupo persiguiendo, por ejemplo, metas orientadas a perfeccionar su presencia e imagen corporal ante el grupo. De este modo, una falta de las necesidades básicas o una frustración de las mismas podría llevar a los estudiantes a desarrollar substitutos de necesidades, la cuales en sí pueden tener consecuencias negativas (e.g., obsesión o ansiedad por la evaluación del físico) que interfieran en el logro de los nutrientes que realmente necesita la persona. No obstante, este estudio, al no medir la satisfacción de las necesidades, no puede mostrar si la relación de la frustración de las necesidades mantiene una asociación más fuerte con consecuencias desadaptativas para la persona (e.g., ansiedad físico-social) que la percepción de la insatisfacción de las necesidades. Futuros estudios podrían considerar utilizar la medida de la satisfacción y frustración de las necesidades al mismo tiempo para ver sus efectos en consecuencias no sólo adaptativas sino también desadaptativas para el desarrollo y bienestar de las personas. De esta manera se podría apoyar la hipótesis que algunos trabajos han sugerido de que la frustración de las necesidades se muestra como un mejor predictor de las conductas desadaptativas que la satisfacción (Barhtolomew, Ntoumanis, Ryan, Bosch, et al., 2011 Bartholomew, Ntoumanis, Ryan, y Thøgersen-Ntoumani, 2011).

En resumen, este estudio ha dado muestras de la validez y fiabilidad de la EFNP en España parar el contexto del ejercicio físico de acuerdo con postulados 
de la TNPB (Deci y Ryan, 2000; Ryan y Deci, 2002). Con esta escala se ofrece una herramienta que permite medir la percepción de los practicantes de ejercicio físico en lo referente a la frustración de sus necesidades psicológicas básicas. Esto permitirá profundizar en la evaluación de los factores contextuales que dentro del ejercicio físico pueden minar el bienestar psicológico, a través de la frustración de los nutrientes necesarios que necesita la persona para su desarrollo.

\section{AGRADECIMIENTOS}

La realización de este trabajo fue posible gracias al proyecto de investigación: «Análisis de la influencia de las clases de Educación Física en la adherencia a la práctica deportiva y la adopción de hábitos de vida saludables tras finalizar la escolarización obligatoria" (Ref. DEP2010-17063), financiado por el Ministerio de Ciencia e Innovación.

\section{REFERENCIAS}

Bartholomew, K., Ntoumanis, N., Ryan, R., Bosch, J., y Thogersen-Ntoumani, C. (2011). Self-Determination theory and diminished functioning: The role of interpersonal control and psychological need thwarting. Personality and Social Psychology Bulletin, 37, 1459-1473.

Bartholomew, K., Ntoumanis, N., Ryan, R., y Thøgersen-Ntoumani, C. (2011). Psychological need thwarting in the sport context: Assessing the darker side of athletic experience. Journal of Sport and Exercise Psychology, 33, 75-102. Bartholomew, K., Ntoumanis, N., y Thøgersen-Ntoumani, C. (2011). Self-Determination theory and the darker side of athletic experience: The role of interpersonal control and need thwarting. Sport and Exercise Psychology Review, 7, 23-27.

Bentler, P. M. (1989). EQS structural equations program manual. Los Angeles: BMDP Statistical Software.

Biddle, S. J. H., y Fox, K. R. (1989). Exercise and health psychology: Emerging relationships. British Journal of Medical Psychology, 62, 205-216.

Brien, M., Forst, J., Mageau, G. A., Boudrias, J. S., Desrumaux, P., Brunet, L., Morin, E. M. (2012). The basic psychological needs at work scale: Measurement invariance between Canada and France. Applied psychology: health and well -being, 4(2), 167-187.

Browne, M. W., y Cudeck, R. 1993. "Alternative ways of assessing model Fit". En K. Bollen y J. Long (Eds.), Testing Structural Equation Models (pp. 136162). Newbury Park, CA: Sage.

Brunet, J. y Sabiston, C. M. (2009). Social physique anxiety and physical activity: A self-determination theory perspective. Psychology of Sport and Exercise, 10, 329-335.

Byrne, B. M. (2001). Structural equation modeling with Amos: Basic concepts, applications, and programming. Mahwah, NJ: Erlbaum. 
Cheung, G. W., y Rensvold, R. B. (2002). Evaluating goodness-of-fit indexes for testing measurement invariance. Structural Equation Modeling, 9, 233-255.

Deci, E. L., y Ryan, R. M. (1985). Intrinsic motivation and self-determination in human behavior. New York: Plenum.

Deci, E. L., y Ryan, R. M. (2000). The "what" and "why" of goal pursuits: Human needs and the self-determination of behavior. Psychological Inquiry, 11, 227-268.

Gagné, M., Ryan, R. M., y Bargmann, K. (2003). Autonomy Support and need satisfaction in the motivation and well-being of gymnasts. Journal of Applied Sport Psychology, 15, 372-390.

Gargari, B. P., Khadem-Haghighian, M., Taklifi, E., Hamed-Behzad, M., y Shahraki, M. (2010). Eating attitudes, self-esteem and social physique anxiety among Iranian females who participate in fitness programs. Journal of Sports Medicine and Physical Fitness, 50, 79-84.

Glenister, D. (1996). Exercise and mental health: A review. The Journal of the Royal Society for the Promotion of Health, 116, 7-13. doi:10.1177/146642409611600102

Gillet, N., Fouquereau, E., Forest, J., Brunault, P., y Colombat, P. (2011). The impact of organizational factors on psychological needs and their relations with well-being. Journal of Business and Psychology, Online First ${ }^{T M}$, doi:10.1007/ s10869-011-9253-2

Hambleton, R. K. (1996). Adaptación de test para su uso en diferentes idiomas y culturas: fuentes de error, posibles soluciones y directrices prácticas. En J. Muñiz (Ed.), Psicometría, 207-238. Madrid: Universitas.

Hausenblas, H. A., y Symons Downs, D. (2002). Exercise dependence: A systematic review. Psychology of Sport and Exercise, 3, 89-123. doi:10.1016/ s1469-0292(00)00015-7

Hodge, K., Lonsdale, C., y Ng, J. Y. Y. (2008). Burnout in elite rugby: Relationships with basic psychological needs fulfillment. Journal of Sports Sciences, 26, 835-844.

Hu, L., y Bentler, P. M. (1999). Cutoff criteria for fit indexes in covariance structure analysis: Conventional criteria versus new alternatives. Structural Equation Modeling, 6, 1-55. doi:10.1080/10705519909540118

Jöreskog, K. G., y Sörbom, D. (1993). LISREL 8: Structural equation modeling with the SIMPLIS command language. Chicago: Scientific Software.

Luján, J. F., y Ferriol, A. G. (2011). Escala de percepción de promoción del bienestar para entrenadores (EPPBE): Análisis inicial de sus propiedades psicométricas y validez. Revista Internacional de Ciencias del Deporte, 7, 393-407.

Lynn, M. R. (1986). Determination and quantification of content validity. Nursing Research, 35, 382-385.

Mallinson, S. H., y Hill, A. P. (2011). The relationship between multidimensional perfectionism and psychological need thwarting in junior sports participants. Psychology of Sport and Exercise, 12, 676-684. 
Motl, R.W., y Conroy, D. E. (2000). Validity and factorial invariance of the Social Physique Anxiety Scale. Medicine and Science in Sports and Exercise, 5, 1007-1017.

Motl, R.W., y Conroy, D. E. (2001). The Social Physique Anxiety Scale: Cross validation, factorial invariance, and latent mean structure. Measurement in Physical Education and Exercise Science, 5, 81-95. doi:10.1207/ s15327841mpee0502_2

Moreno-Murcia, J. A., Huéscar, E., y Cervelló, E. (2012). Prediction of adolescents doing physical activity after completing secondary education. Spanish Journal of Psychology, 15, 90-100.

Moustaka, F. C., Vlachopoulos, S. P., Kabitsis, C., y Theodorakis, Y. (2012). Effects of an autonomy-supportive exercise instructing style on exercise motivation, psychological well-being, and exercise attendance in middle-age women. Journal of Physical Activity and Health, 9, 138-150.

Ntoumanis, N. (2012). A self-determination theory perspective on motivation in sport and physical education: Current trends and possible future research directions. En G. C. Roberts y D. C. Treasure (Eds.), Motivation in sport and exercise: Volumen 3 (pp. 91-128). Champaign, IL: Human Kinetics.

Pyszczynski, T., Greenberg, J., y Solomon, S. (2000). Why do we need what we need? A terror management perspective on the roots of human social motivation. Journal of Psychological Inquiry, 8, 1-20.

Reis, H. T., Sheldon, K. M., Gable, S. L., Roscoe, J.,y Ryan, R. M. (2000). Daily well-being: The role of autonomy, competence, and relatedness. Personality and Social Psychology Bulletin, 26, 419-435.

Ryan, R. M. (1995). Psychological needs and the facilitation of integrative processes. Journal of Personality, 63, 397-427.

Ryan, R. M., y Deci, E. L. (2000). The darker and brighter sides of human existence: Basic psychological needs as a unifying concept. Psychological Inquiry, 11, 319-338.

Ryan, R. M., y Deci, E. L. (2002). Overview of self-determination theory: An organismic dialectical perspective. En E. L. Deci y R. M. Ryan (Eds.), Handbook of self-determination research (pp. 3-33). Rochester, NY: University of Rochester Press.

Sáenz-Alvarez, P., Sicilia, A., González-Cutre, D., y Ferriz, R. (en prensa). Psychometric properties of the Social Physique Anxiety (SPAS-7) in Spanish adolescent. The Spanish Journal of Psychology.

Salmon, P. (2001). Effects of physical exercise on anxiety, depression, and sensitivity to stress: a unifying theory. Clinical Psychology Review, 21, 33-61. doi: 10.1016/S0272-7358(99)00032-X.

Sallis, J. F., y Owen, N. (1999). Physical Activity and Behavioral Medicine. Thousand Oaks, CA: Sage.

Schumacker, R. E., y Lomax, R. G. (1996). A beginner's guide to structural equation modeling. Mahwah, NJ: Erlbaum.

Thøgersen-Ntoumani, C., y Ntoumanis, N. (2007). A self-determination theory 
approach to the study of body image concerns, self-presentation and selfperceptions in a sample of aerobic instructors. Journal of Health Psychology, 12, 301-315. doi:10.1177/1359105307074267

Thøgersen-Ntoumani, C., Ntoumanis, N., Cumming, J., y Chatzisarantis, N. L. D. (2011). When feeling attractive matters too much to women: A process underpinning the relation between psychological need satisfaction and unhealthy weight control behaviors. Motivation and Emotion, 35, 413-422.

Van den Broeck, A., Vansteenkiste, M., De Witte, H., Soenens, B., y Lens, W. (2010). Capturing autonomy, competence, and relatedness at work: Construction and initial validation of the work-related basic need satisfaction scale. Journal of Occupational and Organizational Psychology, 83(4), 9811002.

Vlachopoulos, S. P., y Michailidou, S. (2006). Development and initial validation of a measure of autonomy, competence, and relatedness in exercise: The basic psychological needs in exercise scale. Measurement in Physical Education and Exercise Science, 10(3), 179-201.

Wilson, P. M., Longley, K., Muon, S., Rodgers, W. M., y Murray, T. C. (2006). Examining the contributions of perceived psychological need satisfaction to well-being in exercise. Journal of Applied Biobehavioral Research, 11, 243264. 


\section{APÉNDICE.}

Escala de Frustración de las Necesidades Psicológicas en el Ejercicio Físico

\section{Frustración necesidad de autonomía}

1. Me siento impedido/a para tomar decisiones con respecto al ejercicio físico que realizo

4. Me siento presionada/o a comportarme de una cierta manera

7. Me siento obligada/o a seguir las decisiones de los demás

10. Me siento presionada/o a asumir el ejercicio físico planeado

\section{Frustración necesidad de competencia}

2. Hay ocasiones en las que me siento incompetente porque los demás se hacen expectativas poco realistas de mí

5. Hay veces en las que me han dicho cosas que me hacen sentir incompetente

8. Hay situaciones donde me siento incapaz

11. Me siento incompetente porque no me dan la oportunidad de desarrollar mi potencial

\section{Frustración necesidad de relación}

3. Me siento rechazado/a por los que me rodean

6. Siento que otros pueden tener una actitud despectiva hacia mí

9. Siento que no le gusto a otras personas

12.Percibo que algunos de los compañeros/as con los que me rodeo sienten envidia cuándo tengo éxito 